\title{
MUTATION OF THE KILLER CYTOPLASMIC FACTOR IN PARAMECIUM AURELIA
}

\author{
RUTH V. DIPPELL \\ Department of Zoology, Indiana University, Bloomington, Indiana *
}

Received 16. ii.5o

Sonneborn (1939, 1943) has shown that certain strains of Paramecium aurelia, known as " killers," liberate into the medium in which they live a poisonous substance called "paramecin." The latter has no effect upon the animals which produce it, but other strains of paramecia, known as "sensitives," are killed by its action. The killer trait was found by Sonneborn to be cytoplasmically controlled by "kappa," a self-multiplying, visible particle of which several hundred are present in the cytoplasm of each killer animal (Preer, 1946, 1948b; Sonneborn, 1946). These bodies bear a striking resemblance to the nuclear genes in their ability to control an hereditary trait and in their power of self-duplication. However, one of the fundamental properties exhibited by nuclear genes, that of mutability, has, until the present study, been reported for cytoplasmic determinants of heredity in only two cases : mutation of plastids in higher plants (see review by Rhoades, 1946) and mutation of the genoid controlling $\mathrm{CO}_{2}$ sensitivity in Drosophila (Goldstein, 1949). In 1948, this writer published a preliminary report of mutations of kappa. Sonneborn (1943) and Preer (1948a) had reported what they believed to be indications of a kappa mutation, but the cultures in question were never analysed. The present paper gives in detail the analysis demonstrating that mutation of kappa and not of the $K$ gene provides the physical basis for five observed changes or mutations of the killer character. A brief account is included of the origins of the mutant stocks, the diverse types of killing they manifest, and the nature of the kappas present in the mutants.

\section{THE MUTANT KILLER STOCKS}

\section{(a) Detection and maintenance}

The paramecin produced by each killer stock causes sensitives to undergo a series of characteristic changes leading to death of the affected animals (Sonneborn, 1939, 1943, 1946 ; Preer, 1948a). The present paper deals with the paramecins produced by two killer stocks,

* Contribution No. 428 from the Department. Submitted to the Faculty of the Graduate School in partial fulfilment of the requirements for the degree Doctor of Philosophy in the Department. This investigation was supported by Indiana University and Rockefeller Foundation grants in aid of research on Protozoan Genetics at Indiana University.

The author was a Fellow of the American Association of University Women during part of the period over which this investigation extended. 
47 and 5 I, of variety 4 . The typical pre-lethal stages caused by the paramecin of killer stock $5^{\mathrm{I}}$ are shown in plate I, figs. $(a)-(e)$. Stage $(a)$ is recognisable about two hours after exposure and stage $(e)$ about twelve hours after exposure of sensitives to stock $5^{\mathrm{I}}$ paramecin at $27^{\circ} \mathrm{C}$. However, the time at which the first morphological changes become apparent and the duration of the various stages are dependent upon a number of factors, e.g. temperature and nutritive conditions of both killer and sensitive animals. The characteristic pre-lethal stage caused by killer stock 47 is development of a large vacuole in the posterior part of the body (plate I, fig. $(f)$ ).

Changes in the killer character of stocks $5 \mathrm{I}$ and 47 were regarded as mutations if they remained permanently heritable throughout vegetative and sexual reproduction. The mutants differed from the original killers either in the type of pre-lethal stages induced in sensitives or in the strength of killing as measured by the number of sensitive animals killed in a mixture with a given number of killers in a standard time interval.

Five different sensitive stocks were employed initially as detectors of the type of killing, but the most sensitive of these, stock 31 of variety 8 , was used in most of the tests.

Whenever a culture displayed a mutant type of killing, some animals were individually isolated and grown at maximal rate, while others were isolated and grown at an average rate of one fission per day. The necessity for maintaining the isolations at different rates of growth is pointed out on p. I68. After each single animal isolation had given rise, by repeated fission, to a culture containing approximately 1000 animals, a sample of the culture was tested for its ability to kill and for its type of killing. Mutant killer clones thus obtained were carried as stock cultures, being given each day enough standard culture medium to permit an average growth rate of one fission per day. At approximately ten-day intervals new subcultures were started either as single animal isolations or as mass subcultures. Each new subculture was routinely checked for its killer character.

Successive mutants obtained from a given killer stock are designated as $m \mathrm{I}, m 2$, etc., following the stock number $(47$ or $5 \mathrm{I})$. In a preliminary paper (Dippell, I948), the four mutants of stock 51 were referred to as the "first" to "fourth" mutants; these correspond to the mutants $5^{1} m_{1}$ to $5^{1} m_{4}$ of the present paper, except that $5^{1} m_{1}$ and $5^{1} m 2$ are here reversed (i.e. the "first" mutant of the earlier paper is here designated $5^{1} \mathrm{~m} 2$ ). The change manifested by the culture designated as $5 \mathrm{I} m 4$ proved transient; as it was not a true mutation, $5 \mathrm{I} m 4$ is not dealt with further in this paper.

(b) Origin of the mutant stocks

Of the five mutations analysed in this study, all arose spontaneously in so far as known.

Origin of $5^{\mathrm{I} m \mathrm{I}}$ and $5^{\mathrm{I} m 2 .--R e s e r v e ~ c u l t u r e s}$ of each stock are 
maintained in this laboratory in duplicate bottles fed once every three weeks. The duplicate bottles of stock $5^{1}$ were subcultured from a common source in 1946 and in the following year one of these bottles was found to contain $5 \mathrm{I} \mathrm{m} \mathrm{I}$, the other, $5 \mathrm{I} \mathrm{m} 2$.

Origin of $5 \mathrm{r} m 3$.- This mutant was found by Preer in a culture of stock $5^{\mathrm{I}}$ possessed by J. A. Harrison of Temple University and obtained originally from Sonneborn.

Origin of $5 \mathrm{rm} 5$.-A single animal isolated from a typical culture of stock $5 \mathrm{I}$ produced descendants with markedly reduced killing power. One of the descendants was isolated and yielded the mutant culture $5^{\mathrm{I} m} 5$.

Origin of $47 \mathrm{mr}$. - In our laboratory, stock 47 lost the killer character during a period of several years when it was maintained in stock bottles but not employed for experiments. While it was still a killer, a sample of the stock was sent to Father J. A. Frisch of Canisius College. In 1946 , he returned a subculture to Sonneborn's laboratory. This was immediately found to be characterised by a type of killing identical with that of typical stock $5 \mathrm{I}$. However, Father Frisch never possessed stock $5 \mathrm{I}$. The fact that $47 \mathrm{~m} \mathrm{I}$ was derived from stock 47 and not from stock $5 \mathrm{I}$ was, moreover, demonstrated by breeding analysis (p. 173). (This same type of analysis showed that the remaining mutants were derived from stock 5 r.)

\section{(c) The phenotypes of the mutant stocks}

Among the five mutant killers, only two types of killing have thus far been found. The first type produces changes in sensitives which appear to be identical with those produced by $5^{\mathrm{I}}$ paramecin and will be referred to as $5^{\mathrm{I}}$ killing. The second type, discovered in $5 \mathrm{r} \mathrm{m} \mathrm{r}$, differs strikingly from $5^{\mathrm{I}}$ and partially resembles the killing action of stock $G$ (variety 2) described by Sonneborn (1939) and more extensively by Preer (1948a). This type of killing is known as "spinner" or "Sp." Sensitive animals exposed to $5 \mathrm{I} \mathrm{mI}$ paramecin reverse their normal direction of rotation and subsequently increase the speed of this rotation. As this is accompanied by little or no forward movement, it results in violent spinning of the sensitives on their longitudinal axes. In later stages, animals commoniy develop one or two large vacuoles within the body, form along the aboral body surface a huge blister into which the body contents subsequently empty, or retain a somewhat normal appearance but settle to the bottom and become almost completely paralysed before death (plate I, figs. $(g),(h)$ and $(i))$. This type of killing progresses to completion more rapidly than that of 5 I or $G$.

Of the five mutants thus far analysed, one $(5 \mathrm{I} \mathrm{mI})$ produces only the Sp type of killing and one $\left(5^{\mathrm{I}} \mathrm{m}_{5}\right)$ produces only the $5^{\mathrm{I}}$ type of killing. The mutant stocks $5^{1} \mathrm{~m} 2$ and $5_{1} \mathrm{~m} 3$ are mixed with respect to the two types of killing : cultures derived from single animals of these mutant stocks kill some sensitives in one way and some animals of the same 
sensitive stock in the other way. Mutant $47 \mathrm{~m} \mathrm{r}$ was first believed to manifest only the $5 \mathrm{I}$ type of killing but, following several months of culture, a small amount of Sp killing was also detected.

Some of these mutants are also distinguished in other ways. Thus, $5^{\text {I } m_{5}}$ kills very weakly (see p. I 7 ), often not at all, and mutants manifesting the Sp type of killing can retain this type of killing only when grown less rapidly than three fissions per day at $27^{\circ} \mathrm{C}$.

Finally, the mutants are characterised by their resistance to the two types of paramecin : in brief, each mutant is resistant to the type or types of paramecin it produces. Thus, $5 \mathrm{r} m \mathrm{r}$, which produces only $\mathrm{Sp}$ paramecin, is resistant to this type only and is sensitive to $5^{\mathrm{I}}$ paramecin. The reverse is true for $5 \mathrm{I} \mathrm{m}_{5}$. The remaining mutants, producing both types of paramecin, are resistant to both. In agreement with these relations, table I shows the complex results observed when mixtures are made between any two kinds of killers.

TABLE I

Results obtained when living animals of stock $5^{I}$, the mutant killer stocks and sensitive stock $3^{I}$ are mixed together in all possible combinations of two each. - indicates no killing, $5^{\mathrm{I}}$ indicates $5^{\mathrm{I}}$ type of killing, $\mathrm{Sp}$ indicates spinner type of killing, and $-5 \mathrm{I}$ indicates extremely weak and sporadic $5^{I}$ type of killing. Each record represents a composite result of six separate tests carried out within a six-month period.

\begin{tabular}{|c|c|c|c|c|c|c|c|}
\hline \multirow{3}{*}{$\begin{array}{l}\text { Living } \\
\text { animals }\end{array}$} & \multicolumn{7}{|c|}{ Living animals } \\
\hline & \multirow{2}{*}{$\begin{array}{c}\text { Original } \\
\text { killer } \\
\text { stock } \\
5 \mathrm{I}\end{array}$} & \multicolumn{5}{|c|}{ Mutant killer stocks } & \multirow{2}{*}{$\begin{array}{l}\text { Sensitive } \\
\text { stock } \\
3^{1}\end{array}$} \\
\hline & & $5^{\mathrm{I} m \mathrm{I}}$ & $5^{1 m 2}$ & $5^{1 m 3}$ & $5^{1 m_{5}}$ & $47 \mathrm{mi}$ & \\
\hline $5^{1}$ & - & $5^{x}, \mathrm{Sp}$ & $\mathrm{Sp}$ & Sp & - & Sp & \\
\hline $5 \operatorname{ImI}$ & $5^{1}, \mathrm{Sp}$ & -2 & $-5^{1}$ & $5^{1}$ & $-5 \mathrm{x}, \mathrm{Sp}$ & $5^{1}$ & $\mathrm{Sp}$ \\
\hline $51 m 2$ & $\mathrm{Sp}$ & $-5^{x}$ & - & - & $\mathrm{sp}$ & - & $-51, S p$ \\
\hline $51 m_{3}$ & $\mathrm{Sp}_{\mathrm{p}}$ & $5^{1}$ & - & - & $\mathrm{Sp}$ & - & $5^{x}, \mathrm{Sp}^{\mathrm{r}}$ \\
\hline $51 m 5$ & $\frac{1}{\mathrm{sn}}$ & $-5 \mathrm{x}, \mathrm{Sp}$ & Sp & $\mathrm{Sp}$ & $\bar{s}$ & $\mathrm{Sp}$ & -51 \\
\hline $\begin{array}{c}47 m \mathrm{mI} \\
31\end{array}$ & $\begin{array}{l}\mathrm{sp} \\
5^{1}\end{array}$ & $\stackrel{51}{S p}$ & $-5^{\prime}, \mathrm{Sp}$ & ${ }_{51}, \mathrm{Sp}$ & $\begin{array}{l}\text { Sp } \\
-5 I\end{array}$ & ${ }_{5 x}, \overline{S p}$ & $5^{x}, S p$ \\
\hline
\end{tabular}

Simpler and clearer results (table 2), in complete agreement with the preceding, are obtained by mixing breis of each kind of killer with animals of each kind. Animal-free brei was obtained by repeatedly forcing a concentrated culture of the animals whose killing action was to be studied through a 25 gauge hypodermic needle until no intact paramecia remained (Sonneborn, 1950 $a$ ). Living animals to be tested for resistance or sensitivity to this killer brei were then added to a known volume of the brei. Vegetative animals used in the tests were standardised by growing them in test tubes at an approximate rate of one fission per day for five days prior to testing. Mixtures were observed frequently over a forty-eight hour period. 
From the data in tables $\mathrm{I}$ and 2, one can determine for each stock its type of killing and its resistance or sensitivity to the paramecins

TABLE 2

The results obtained when living animals of stock $5^{I}$, the mutant killer stocks and sensitive stock $3 I$ are mixed in all combinations with breis obtained from those stocks. (Cf. Table I legend for explanation of symbols.) Each record represents a composite of the results of three separate tests conducted within a four-month period.

\begin{tabular}{|c|c|c|c|c|c|c|c|}
\hline \multirow{3}{*}{ Brei } & \multicolumn{7}{|c|}{ Living animals } \\
\hline & \multirow{2}{*}{$\begin{array}{l}\text { Original } \\
\text { killer } \\
\text { stock } 51\end{array}$} & \multicolumn{5}{|c|}{ Mutant killer stocks } & \multirow{2}{*}{$\begin{array}{r}\text { Sensitive } \\
\text { stock } 31\end{array}$} \\
\hline & & $5 \mathrm{I} m \mathrm{I}$ & $5^{1} \mathrm{~m} 2$ & $5_{1} m_{3}$ & $5^{1 m 5}$ & $47 \mathrm{mI}$ & \\
\hline $5^{I}$ & - & $5^{\mathrm{I}}$ & - & - & - & - & $5 \mathrm{I}$ \\
\hline $5 \mathrm{ImI}$ & Sp & - & - & - & Sp & 一 & Sp \\
\hline $51 \mathrm{~m}$ & Sp & -51 & Z & $\overline{-}$ & Sp & - & $\begin{array}{l}-51, \mathrm{Sp} \\
5 \mathrm{I}\end{array}$ \\
\hline 5 I $m 5$ & - & $-5 I$ & - & - & - & - & $-5 \mathrm{I}$ \\
\hline $47 \mathrm{mI}$ & Sp & $5^{I}$ & - & - & Sp & - & $5 \mathrm{I}, \mathrm{Sp}$ \\
\hline $3 I$ & - & - & - & - & - & - & - \\
\hline
\end{tabular}

produced by other stocks. These three aspects of the killer character, when combined, comprise the killer phenotype of a stock and are summarised in table 3 .

TABLE 3

The killer phenotype of the mutants and original $5_{I}$ killer stock. This table shows the type or types of killing action on sensitive stock $3 I$. The strength of the killing reaction is indicated by the number of $(+)$ symbols. Also shown is the resistance $(\mathbf{R})$ or sensitivity $(\mathbf{S})$ of a given stock to the two general types of paramecin found among the mutant killers, viz. $5^{I}$ paramecin associated with $5^{I}$ type of killing and Sp paramecin associated with spinner type of killing.

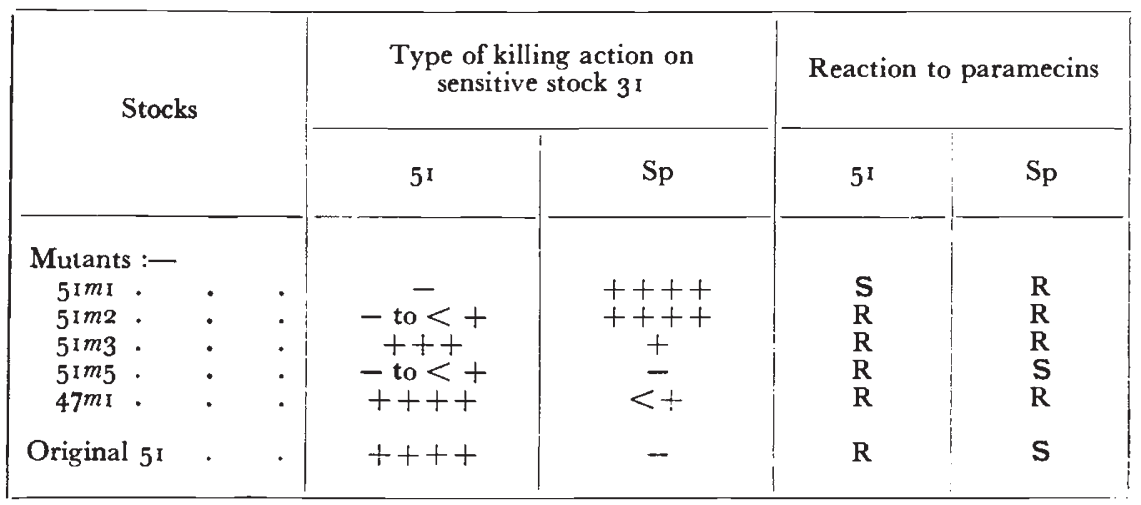

\section{ANALYSIS OF THE KILLER MUTATIONS}

Sonneborn has shown that inheritance of the stock $5 \mathrm{I}$ killer character depends upon two constitutional factors : a cytoplasmic factor, kappa, and certain genes. The principal gene, $K$, is essential 
for the maintenance of kappa but cannot initiate its production. If either $K$ or kappa or both are removed, the killer trait is quickly lost. Another gene, $s$, in the killer stock $5^{\mathrm{I}}$ is also involved, for when this is replaced by $S$ from certain sensitive stocks, kappa may slowly disappear (Sonneborn, 1947b). In view of this knowledge, the change from the original to the mutant killer character could conceivably be due to mutation of $K$ to $K^{\prime}$, controlling some modification of kappa or paramecin; mutation of one or more other loci that could affect kappa or paramecin; or mutation of kappa itself. These three possibilities were investigated by means of the following experiments, the first three of which answer the question of the existence of a gene difference between the mutant and the original 5 I stock with respect to control of the killer character.

\section{(a) Materials}

Special stocks used in the genetic analysis.-In addition to the mutant and original stocks already mentioned, two stocks experimentally obtained by Sonneborn were employed in the genetic analysis. One of these, the " 5 I sensitive" or " $5 \mathrm{IS}$ " stock, was genically identical with killer stock 5 I but lacked kappa and so was sensitive to paramecins produced by $5 \mathrm{I}$ and by other killer stocks. The stock was obtained by freeing 5 I killer animals of kappa, using the method of growth at $33.8^{\circ} \mathrm{C}$. (Sonneborn, I950a).

The second stock used in the analysis, stock $\mathrm{r} 86$, is highly isogenic with killer stock $5 \mathrm{I}$ but contains the recessive $k$ gene from sensitive stock 29 of variety 4 . This stock was obtained by crossing 5 I killers to stock 29 , inducing autogamy in the $F_{1}$ killer cultures, and backcrossing a homozygous recessive to the killers. These steps were repeated through seven successive backcross generations. Stock 186 is one of the recessives extracted after the seventh backcross.

\section{(b) Methods}

Crossing stocks.-The methods employed for making crosses and for avoiding both selfing and cytogamy (double self-fertilisation) were those set forth by Sonneborn (1950a).

Isolation of the $F_{1}$ generation.-Newly formed conjugant pairs were isolated separately into culture medium at $27^{\circ} \mathrm{C}$. After the completion of conjugation, the two members of each conjugant pair were separately isolated and allowed to reproduce by fission, the resulting clones representing the $F_{1}$ generation. (Sensitive animals can successfully be crossed to killers because they are not affected by paramecin during the process of conjugation.)

Culture of the $F_{1}$.-As mentioned on p. 168 , it was necessary to cultivate some of the mutant-type killers at a slow rate of growth in order to maintain their killer character, while still other mutant killers and the original stock 5 I could be grown at maximal rate without loss of the killer phenotype. Two kinds of media were thus employed 
in the culture of the stocks. One type (standard medium) supported a maximal rate of fission and consisted of baked head lettuce medium inoculated with Aerobacter aerogenes (Sonneborn and Dippell, I946). Stocks $5^{\mathrm{I}}$ and $5^{\mathrm{I}} \mathrm{m}_{5}$ were cultured in this medium. The second type of culture fluid, maintaining slow growth, consisted of " exhausted" culture fluid supplemented with standard medium (Dippell's method : Sonneborn, I950a). Mutant stocks $5 \mathrm{I} m \mathrm{I}, 5 \mathrm{I} m 2$ and $5 \mathrm{I} m 3$ were cultured in this fluid. Mutant $47 \mathrm{~m} \mathrm{I}$ was at first cultured in standard medium; later, however, it was found to lose one of its types of killing under these conditions and thereafter was cultivated in "exhausted" culture fluid.

Testing the $F_{1}$ for killing capacity.-To test each exconjugant clone for killing, approximately roo animals of the clone were placed in each of three slide depressions. Approximately roo animals of a killer culture of the same type as used in the cross were added to one depression, (a), and 300 animals of a sensitive culture were added to a second depression, $(b)$. The third depression, $(c)$, consisted only of the line being tested (control). If killing occurred in $(a)$ but not in $(b)$ or $(c)$, the $\mathrm{F}_{\mathbf{1}}$ clone was sensitive. If killing occurred in $(b)$ but not in $(a)$ or $(c)$, the $\mathrm{F}_{1}$ clone was a killer. Autolethality (i.e. killing in $(a),(b)$ and $(c))$ was not observed. Results of tests were recorded both at the end of twenty-four and forty-eight hours in experiments involving the $5 \mathrm{I}, 5 \mathrm{I} m_{5}$ and $47 \mathrm{~m}_{\mathrm{I}}$ killers and at the end of approximately seven and twenty-four hours in experiments involving the $5 \mathrm{I} m \mathrm{I}$, $5^{\mathrm{I}} m 2$ and $5^{\mathrm{I}} \mathrm{m} 3$ killers, since in the latter three stocks killing action proceeded to completion more rapidly than in the first three. These two sets of observations on each test were sufficient to show the type of killing (i.e. the characteristic morphological changes undergone by the sensitives) and to provide an indication of the strength of killing as measured by the numbers of sensitives killed in a standard time interval in relation to the number of killer animals present. All clones except those that proved to be sensitive to the killers used in the cross, were further tested by mixture with the original $5^{\text {I }}$ killers. If such a mixture showed 5 I type killing, the clone being tested was sensitive to $5 \mathrm{I}$ paramecin. Of course, cultures shown by the first test to be Sp mutant killers would also produce mutant killing in the mixtures with 5 I killers.

Maintenance of the $F_{1}$. - The $\mathrm{F}_{1}$ clones were perpetuated in daily isolation culture (Sonneborn, I950 $a$ ) in standard medium. Under these conditions, autogamy is long prevented, thus permitting the heterozygous genotype to persist. This method, which permits reproduction at maximal rate, was unsuitable for killers that had to be grown slowly in order to retain the killer trait. For them, the following method was employed. Instead of daily isolations, daily mass subcultures were made and the cultures were kept at $22^{\circ} \mathrm{C}$. This proved to be the most effective procedure for keeping reproductive rate low and at the same time avoiding autogamy. 
Obtaining the $F_{2}$ generation.-The $\mathrm{F}_{2}$ generation was regularly obtained by inducing autogamy in $F_{1}$ clones according to the methods described by Sonneborn (1950a). Animals in the climax stage of autogamy, as shown by their appearance in living condition and as confirmed by staining parallel samples, were isolated singly and maintained at $27^{\circ} \mathrm{C}$. in the same way as set forth on p. 170 for the culture of the $\mathrm{F}_{1}$ clones.

Testing the $F_{2}$ clones.-When rooo to 2000 animals were present in each culture, the clones were tested for killing in the same manner as the $F_{1}$ cultures. On the basis of the results obtained, a 15 to 20 per cent. sample of the total number of lines tested was selected to hold for retesting after an additional io to 15 fissions. A few clones were retested for a third and fourth time as a check on the adequacy of the first test. Since later tests invariably confirmed the results of the first test, the following account will give the results of the first test only.

\section{(c) Experiment I}

In this experiment, each mutant killer was mated with the $5 \mathrm{I}$ sensitive stock described on p. 170 . This stock, it will be recalled, is genically identical with the killer stock $5^{\mathrm{I}}$ but lacks kappa. $F_{1}$ killers from such a cross therefore should give rise at autogamy to an $F_{2}$ generation in which 50 per cent. of the clones are homozygous for the original $5 \mathrm{I}$ killer gene, $K$, and $5^{\circ}$ per cent. are homozygous for the killer gene in the mutant. If now, the difference between a mutant and the original killer stock is due to a mutation at the $K$ locus, then the $F_{2}$ generation obtained from the $F_{1}$ killer should show segregation into two types of killers, mutant and original. On the other hand, if the mutant killers and original $5^{\mathrm{I}}$ killer are effectively alike in genes controlling the killer character, then no segregation should occur in the $\mathrm{F}_{2}$, and all clones should remain mutant type killers.

As appears in table 4, the results obtained with each of the five mutants show that the mutant killer character is not due to a mutation at the $K$ locus, for all $\mathrm{F}_{2}$ killer clones showed exclusively the mutant type of killing in spite of the fact that half of them must have been homozygous for the original $K$ gene.

It will be noted that fewer $\mathrm{F}_{2}$ clones were tested than were obtained. The discrepancy in the first four crosses is due to (I) failure of some $F_{2}$ isolations to grow, (2) production of extremely weak clones which could not be adequately tested, and (3) the elimination of some clones which proved, by cytological check, to be of vegetative rather than autogamous origin.

The same applies in part to the fifth cross, but in this case there is an additional cause of the discrepancy. The cross of $47 \mathrm{~m} \mathrm{I} \times 5^{\mathrm{IS}}$, unlike the others, is between two stocks originating from diverse sources in nature. Such inter-stock crossing invariably yields high mortality among the $F_{2}$ clones in contrast to nearly roo per cent. 
viability of the $F_{2}$ generation obtained from crosses between individuals descended from a common ancestor. (The high percentage of $F_{2}$ mortality obtained when $47 \mathrm{mI}$ was crossed to stock $5 \mathrm{I}$ and the absence

TABLE 4

Summary of the results of crossing mutant killers to the $5 I$ sensitive stock. Symbols: $\mathrm{K}=$ killer; $\mathrm{MK}=$ mutant killer of type used in cross; Or. $\mathrm{K}=$ original unmutated killer; $\mathrm{S}=$ sensitive. Within each cross, approximately the same number of $F_{2}$ clones was obtained from each $F_{1}$ clone.

\begin{tabular}{|c|c|c|c|c|c|c|c|c|c|c|c|}
\hline \multirow{4}{*}{ Cross } & \multirow{4}{*}{$\begin{array}{l}F_{1}: \text { No. } \\
\text { of clones } \\
\text { used as } \\
\text { source } \\
\text { for } F_{2}\end{array}$} & \multirow{4}{*}{$\begin{array}{l}\mathrm{F}_{2} \text { : No. } \\
\text { of clones } \\
\text { obtained }\end{array}$} & \multicolumn{9}{|c|}{$F_{2}:$ Results } \\
\hline & & & \multicolumn{3}{|c|}{ Observed } & \multicolumn{6}{|c|}{ Theoretical } \\
\hline & & & \multirow{2}{*}{ MK } & \multirow{2}{*}{ Or.K } & \multirow{2}{*}{$\mathrm{S}$} & \multicolumn{3}{|c|}{ 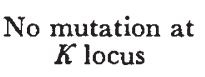 } & \multicolumn{3}{|c|}{$\begin{array}{c}\text { Mutation at } K \\
\text { locus }\end{array}$} \\
\hline & & & & & & MK & Or.K & $\mathrm{S}$ & MK & Or.K & $\mathrm{S}$ \\
\hline${ }^{2} \mathrm{I} m \mathrm{IK} \times 5_{1} \mathrm{~S}$ & 3 & 90 & 88 & o & o & 88 & 0 & 0 & $44 \div 0$ & $44^{\circ} 0$ & 0 \\
\hline $5 \mathrm{Im} m \times{ }^{\mathrm{IS}}$ & $\frac{3}{7}$ & 103 & 102 & o & o & 102 & $o$ & 0 & $51 \cdot 0$ & $51 \cdot 0$ & 0 \\
\hline${ }_{51} \mathrm{~m} 3 \mathrm{~K} \times{ }_{5} \mathrm{IS}$ & 5 & II 9 & II 6 & o & o & II 6 & $o$ & 0 & 58.0 & $58 \cdot 0$ & 0 \\
\hline $5 \mathrm{Im} 5 \mathrm{~K} \times{ }_{5} \mathrm{IS}$ & 6 & 90 & 89 & o & o & 89 & $o$ & 0 & $44 \cdot 5$ & $44 \cdot 5$ & 0 \\
\hline $47 \mathrm{mIK} \times{ }_{5} \mathrm{IS}$ & 3 & 180 & 103 & o & o & 103 & $o$ & 0 & $51 \cdot 5$ & $\begin{array}{l}77.5 \\
51.5\end{array}$ & 0 \\
\hline
\end{tabular}

of such mortality when $47 m \mathrm{I}$ was mated to stock 47 , indicated clearly that the mutant could not have arisen from stock 5 I but must have originated from stock 47 , see p. 167 .)

The question may be raised as to whether the results obtained in the $F_{2}$ of the above cross are comparable with those obtained in the other four matings when two cultures derived from the same stock were crossed. Sonneborn (1943) has shown, however, that the $K$ gene in stock 47 is allelic to the $K$ gene in stock $5 \mathrm{I}$ and capable of supporting the kappa and killer character of the latter. In view of this fact and the desirability of employing the same culture of stock $5^{\mathrm{I}}$ as the parent for all crosses to the mutant stocks, $47 \mathrm{mI}$ was mated to the $5 \mathrm{I}$ stock rather than to the 47 stock.

Experiment I bears on three additional points. First, the observations show that the mutant killers contain a kappa comparable to the kappa of the original killer. The evidence here is parallel to that adduced by Sonneborn (1943, 1945) in the analysis of the original killer : the sensitive mate of a killer animal gives rise to a killer clone only when cytoplasm passes from the killer to the sensitive during conjugation. In each of the five crosses, there were selected for further study some pairs which did not exchange cytoplasm and some pairs which did. In each pair of the first group, one exconjugant produced only sensitive progeny; while in each pair of the second group, both exconjugants produced killer progeny and of the mutant type. Hence, 
mutant killing, like original type killing, depends on a cytoplasmic factor, kappa.

Second, as with the original killers, the genes of the mutant killers are unable to initiate production of kappa. This is shown by the fact that the sensitive mates in the first group described in the preceding paragraph yielded no killer progeny either in the $F_{1}$ or in their $F_{2}$ descendants obtained by autogamy.

Third, certain other observations, together with the demonstration that mutant killing depends upon kappa, indicate that the latter found in the mutant stocks was derived from the kappa of the original type killers. The only alternatives to this conclusion are de novo origin of the kappa of the mutant killers, and infection from an external source. De novo origin seems practically excluded by the fact that kappa has never arisen de novo in any of the many cultures of $K K$ sensitives carried in the laboratory, although some have been under observation for twelve years. It can, of course, be objected that de novo origin is more probable in killers than in sensitives, but there are no observations that suggest this. As to the possibility of infection from a foreign source, the available evidence (Sonneborn, 1948) shows that infection can occur only in the presence of high concentrations of kappa ( $10^{8}$ particles per c.c.), a condition that was never remotely approached, so far as known, in the history of the mutant killers. Moreover, the fact that $K K$ sensitives have never spontaneously acquired kappa argues against infection as it does against de novo origin. It thus seems that the kappa of the mutant killers could hardly have arisen in any other way than by descent from the kappa of the ancestral original type of killer.

\section{(d) Experiment 2}

The genic identity between the mutants and original stock $5 \mathrm{I}$ with respect to control of the killer trait was considered in experiment 2 by arranging the crosses so as to follow the original $5^{\text {I-type killing }}$ rather than the mutant-type killing analysed in experiment $\mathrm{I}$. Stock $5^{\text {I }}$ killers were crossed to mutant killers or to sensitives derived from mutant killers by freeing the latter of kappa in the manner mentioned on p. I79. Each sensitive obtained in this way thus retained all of the genes possessed by the mutant killer from which it was derived. Pairs showing no cytoplasmic exchange comprised the $F_{1}$ generation. The 5 I killer member of each pair was used to provide an $\mathrm{F}_{2}$ generation by autogamy.

If a gene, and not a kappa, difference determined the difference between the mutants and the original $5 \mathrm{I}$ killer, then that half of the $\mathrm{F}_{2}$ homozygous for the killer gene of the mutant should produce mutant killing, and the remaining $F_{2}$, homozygous for the killer gene from stock $5 \mathrm{I}$, should continue to maintain $5^{\mathrm{I}}$ killing. On the other hand, if the gene controlling the killer characters of the mutants and 
original stock $5^{1}$ are effectively identical, then no segregation should occur in the $F_{2}$, and all of the $F_{2}$ clones derived from the $F_{1} 5^{I-t y p e}$ killer should show $5^{\mathrm{I}}$ type of killing.

The results are summarised in table 5. In the first two crosses, $5^{1}$ killers were crossed to sensitives derived from killer mutants $5^{1} \mathrm{~m} 2$ and $5_{1} m_{5}$. In the case of $51 m_{3}$, however, this mutant killer was crossed directly to 51 killers, due to loss of the derived 5 I $m 3$ sensitive as a result of poor cultural conditions. Mutant killers $5 \mathrm{I} m \mathrm{I}$ and $47 \mathrm{mI}$ were also directly crossed to $5^{\mathrm{I}}$ killers, since, at this time, adequate experimental techniques had not been devised to free these mutants of kappa.

TABLE 5

Summary of results of crossing mutant sensitives and killers to original stock ${ }_{5}$ killers (see table 4 for legend)

\begin{tabular}{|c|c|c|c|c|c|c|c|c|c|c|c|}
\hline \multirow{4}{*}{ Cross } & \multirow{4}{*}{$\begin{array}{l}\mathrm{FI}_{\mathrm{I}} \text { : No. } \\
\text { of clones } \\
\text { used as } \\
\text { source } \\
\text { for } \mathrm{F}_{2}\end{array}$} & \multirow{4}{*}{$\begin{array}{l}\mathrm{F}_{2} ; \text { No. } \\
\text { of clones } \\
\text { obtained }\end{array}$} & \multicolumn{9}{|c|}{$\mathrm{F}_{2}:$ Results } \\
\hline & & & \multicolumn{3}{|c|}{ Observed } & \multicolumn{6}{|c|}{ Theoretical } \\
\hline & & & \multirow{2}{*}{ MK } & \multirow{2}{*}{ Or.K } & \multirow{2}{*}{$S$} & \multicolumn{3}{|c|}{$\begin{array}{l}\text { No mutation at } \\
K \text { locus }\end{array}$} & \multicolumn{3}{|c|}{$\begin{array}{c}\text { Mutation at } K \\
\text { locus }\end{array}$} \\
\hline & & & & & & MK & Or.K & $\mathrm{s}$ & MK & Or.K & $\mathrm{S}$ \\
\hline $5_{5} \mathrm{Im} S \times{ }_{5}^{1 K}$ & 4 & $6 o$ & o & 60 & o & $o$ & $6 o$ & $o$ & $30 \cdot 0$ & $30 \cdot 0$ & $o$ \\
\hline${ }_{5 \mathrm{Im}} \mathrm{S} \times 5_{\mathrm{IK}}$ & 6 & 90 & o & 89 & o & $o$ & 89 & $o$ & 44.5 & $44: 5$ & $o$ \\
\hline $5 \mathrm{ImIK} \times 5 \mathrm{IK}$ & 3 & 90 & o & 88 & o & 0 & 88 & $o$ & $44^{\circ} \circ$ & $44^{\circ} \mathrm{O}$ & $o$ \\
\hline $5^{\mathrm{I} m} \mathrm{~m}_{3} \mathrm{~K} \times 5^{\mathrm{IK}}$ & 2 & 90 & 0 & 89 & o & $o$ & 89 & $o$ & $44 \cdot 5$ & $44 \cdot 5$ & $o$ \\
\hline $47 \mathrm{mIK} \times 5 \mathrm{IK}$ & 2 & 120 & 0 & 69 & o & $o$ & 69 & $o$ & 34.5 & 34.5 & $o$ \\
\hline
\end{tabular}

Whether or not the $F_{2}$ clones were derived from $F_{1} 5^{1-t y p e ~ k i l l e r s ~}$ of a mutant killer $\times 5^{1}$ killer cross or from a similar $F_{1}$ obtained from a mutant sensitive $\times 5^{1}$ killer cross made no difference in the analysis; both types of crosses yielded strictly comparable results, as shown by the table. All $F_{2}$ cultures derived from $F_{1}$ 5I-type killers were themselves $5^{\mathrm{I}}$ killers, although 50 per cent. of the $\mathrm{F}_{2}$ clones were homozygous for the killer gene from the mutant stock. The $5^{1}$ killer character thus can be maintained by the killer gene of each mutant stock, in the absence of the $K$ gene derived from the $5^{1}$ killer parent.

Experiment 2 provided a second important result. In the $5^{\mathrm{I} m \mathrm{IK}} \times 5^{\mathrm{IK}}$ cross, pairs undergoing cytoplasmic exchange were obtained and, although these were not employed as source material for the $\mathrm{F}_{2}$ generation recorded in table 5, they were retained in order to observe the effect of the "mixed" cytoplasm upon the killer phenotype of the resulting $F_{1}$ clones. Exconjugants from five such pairs were grown slowly and tested for type of killing after nine fissions. An equal number of controls showing no cytoplasmic exchange were 
similarly treated. It was found that each exconjugant of those pairs showing no transfer of cytoplasm retained its own type of killing, but both exconjugant members of those pairs exhibiting cytoplasmic exchange became "mixed" with respect to type of killing action. Animals derived from such exconjugants were capable singly of producing two kinds of paramecin, $5 \mathrm{I}$ and $5 \mathrm{I} \mathrm{mI}$. The $\mathrm{F}_{2}$ obtained by autogamy in such " mixed" clones, continued to exhibit mixed killing, although all were roo per cent. homozygous for all their genes. With respect to the alleles at the $K$ locus, half were homozygous for the $K$ gene from $5^{\mathrm{I}} \mathrm{m} \mathrm{I}$ and half were homozygous for the $5^{\mathrm{I}} K$ gene. This result not only shows that mixed killing occurs in entirely homozygous material, but suggests that the mutant killer probably contains mutant types of kappa capable of multiplying and maintaining their character even when associated with original kappa in the same animal. Similar experiments using the $5^{\mathrm{I}} \mathrm{m} 2$ and $5^{\mathrm{I} m} \mathrm{~m}$ mutants in crosses to the $5 \mathrm{I}$ killer were begun but not completed. The only tests carried out were on $\mathrm{F}_{1}$ clones, at approximately the eighth fission; an $\mathrm{F}_{2}$ generation was not obtained. In so far as these experiments progressed, however, they yielded results comparable to those of the $5^{\mathrm{I} m \mathrm{I} K} \times 5^{\mathrm{I} K}$ cross.

\section{(e) Experiment 3}

The preceding experiments give no information as to whether the $K$ gene is essential for the maintenance of the mutant killer traits, as it is for the original killer trait, since all of the stocks thus far employed have been homozygous for this gene. Conceivably, the kappas in the mutant killers might have become independent of nuclear gene control, resulting in the altered type of killing. Although improbable, the possibility was directly tested by breeding experiments replacing the $K$ gene of the mutants with $k$, which, in homozygous condition, does not permit the original kappa to persist and multiply (Sonneborn, 1943).

The source of the $k$ gene in the present experiment was stock 186 which is otherwise largely isogenic with stock $5^{\mathrm{I}}$ (see p. I70). Each of the five mutants was crossed to stock 186 and the $F_{1}$ killers were used to get $\mathrm{F}_{2}$ generations by autogamy. Theoretically, half of the $\mathrm{F}_{2}$ should be homozygous for $K$ and should show the mutant killer character. The other half of the $\mathrm{F}_{2}$ should be homozygous for $k$ and should also yield mutant killer clones if the mutant killer trait is independent of the genes, but should yield sensitive clones if the mutant killer, like the original killer trait, depends on gene $K$.

The results, with two exceptions to be dealt with later, are summarised in table 6 . In each of the five crosses, the $F_{2}$ generation obtained from $\mathrm{F}_{1}$ killers by autogamy consisted of mutant killers and sensitives in the I : I ratio required for a single gene difference. Although the number of $F_{2}$ clones obtained from each $F_{1}$ culture 
was small, the results (with the above exceptions) were in complete agreement with a I: I ratio. Hence, the mutant killer, like the original killer character, disappears in the absence of gene $K$.

TABILE 6

Summary of results of crossing mutant killers to the recessive $\mathrm{kk}$ stock 186

\begin{tabular}{|c|c|c|c|c|c|c|}
\hline \multirow{3}{*}{ Parents } & \multirow{3}{*}{$\begin{array}{c}\text { No. } F_{1} K \\
\text { clones } \\
\text { used to } \\
\text { obtain } F_{2}\end{array}$} & \multirow{3}{*}{$\begin{array}{l}\text { No. } F_{2} \\
\text { clones } \\
\text { obtained }\end{array}$} & \multicolumn{4}{|c|}{ Results of tests on $F_{2}$ clones } \\
\hline & & & \multicolumn{2}{|c|}{ Observed } & \multicolumn{2}{|c|}{ Theoretical } \\
\hline & & & No. $\mathrm{K}$ & No. S & No. $\mathrm{K}$ & No. S \\
\hline 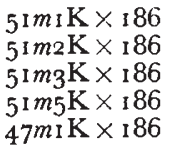 & $\begin{array}{l}5 \\
8 \\
4 \\
3 \\
3\end{array}$ & $\begin{array}{r}90 \\
120 \\
\text { I } 8 \\
90 \\
180\end{array}$ & $\begin{array}{l}40 \\
59 \\
63 \\
43 \\
48\end{array}$ & $\begin{array}{l}46 \\
57 \\
54 \\
47 \\
43\end{array}$ & $\begin{array}{l}43 \cdot 0 \\
58 \cdot 0 \\
58 \cdot 5 \\
45 \cdot 0 \\
45 \cdot 5\end{array}$ & $\begin{array}{l}43 \cdot 0 \\
58 \cdot 0 \\
5^{8 \cdot 5} \\
45^{\circ} 0 \\
45 \cdot 5\end{array}$ \\
\hline Total & 23 & 598 & 253 & 247 & $250 \cdot 0$ & $250 \cdot 0$ \\
\hline
\end{tabular}

Additional confirmation was obtained by other crosses. Thus, three of the $F_{2}$ killers from the cross involving $5^{\text {I } m} 5$ were backcrossed to stock 186 , and autogamy was induced in the backcross killers. These exautogamous clones included 73 killers and 80 sensitives, in agreement with the expected I : I ratio. Further, 6 of the $F_{2}$ sensitives from the same cross $\left(5 \mathrm{I} m_{5} \times\right.$ I 86$)$ were backcrossed to 5 I $m_{5}$ and autogamy was again induced. The exautogamous progeny included 66 killers and $7 \mathrm{I}$ sensitives.

As mentioned above, two groups of $F_{2}$ clones were omitted from table 6 because the ratios obtained differed from all the others. The first was a group of 15 clones obtained from one $F_{1}$ culture from the

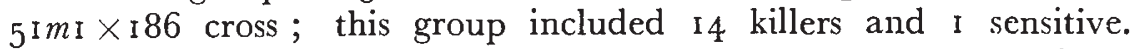
The probability of such a deviation from I : I is about $0 \cdot 00 \mathrm{I}$. Such a result could be formally explained as due to the occurrence of macronuclear regeneration (Sonneborn, I940) at the autogamy giving rise to this $F_{2}$ group, but such an explanation seems unlikely, for macronuclear regeneration is rare under conditions of these experiments. Since the $F_{1}$ culture was discarded before the aberrant $F_{2}$ results appeared, it was impossible to analyse the matter further, and no satisfactory explanation can be given. However, the $F_{1}$ culture from the other exconjugant of the same pair was also a killer and, fortunately, was retained. Therefore, this culture and another $F_{1}$ killer culture (which gave a normal ratio in the $F_{2}$ ) were backcrossed to stock 186 . The two members of each backcross pair were separated at completion of conjugation, grown and tested before the occurrence of autogamy.

In such backcrosses, if no cytoplasmic exchange occurs between 
mates, half of the total number of pairs should yield one sensitive and one killer member in each pair, and the remaining half should show both members to be sensitive.

In each of the two backcrosses, 16 pairs were obtained which showed no exchange of cytoplasm between mates. One backcross group yielded 7 killer-sensitive pairs and 9 sensitive-sensitive pairs; the other produced 6 killer-sensitive pairs and 9 sensitive-sensitive pairs (I pair was lost). Although these groups are small, the results are in close agreement with the expected I : I ratio. Thus, the sister culture of the one that had given the aberrant $F_{2}$ ratio, did not itself give any aberrancy, either in the original $F_{2}$ or in the backcross, the total for the two diverse segregations being $18:$ i3. The one member of this $F_{1}$ pair hence was clearly heterozygous, whatever may have been the peculiarity of the other member of the pair.

The second exceptional ratio was obtained in the cross of $47 \mathrm{mI} \times \mathrm{I} 86$. From one $F_{1}$ clone, $26 F_{2}$ killers and $9 F_{2}$ sensitives were obtained by autogamy. The probability of such a deviation from $I: I$ is about 0.006 . As in the first exception, no explanation can be given, and again, since the $F_{1}$ was discarded after obtaining the $F_{2}$ isolations and before testing of the latter, the cross could not be repeated with the same material. Although the $\mathrm{F}_{2}$ mortality was high (as is to be expected from this cross, see p. I73), the discrepancy could not be due to differential mortality, since other $F_{2}$ groups from the same cross yielded normal I : I ratios in spite of a similarly high incidence of death. Further evidence that normal segregation ratios ordinarily result from this type of cross was obtained by backcrossing one of the $F_{2}$ killers from a normal group to the recessive parent stock 186 and obtaining from the backcross a new generation by autogamy. This generation included 54 killers and 50 sensitives.

Although the two exceptional results remain unexplained, it is clear that ordinarily the data agree closely with the ratio required for segregation of a single pair of alleles and thus leave no doubt either of the dependence of the kappas in the mutants on gene $K$, or of the fact that mutant killers differ from stock 186 at only one effective locus, the $K$ locus, just as does the original killer stock $5 \mathrm{I}$.

Summarising the results of the genetic analysis, it has been shown by experiments $I$ and 2 that the killer mutations cannot be due to a mutation at the $K$ locus. Further, the new characters cannot be the result of mutation at any other locus, for this would also have been detected by $F_{2}$ segregation in experiments $I$ and 2 (which did not take place) and by more complex ratios in experiment 3 (which were not obtained). The two exceptions found in experiment 3 could scarcely be considered support for another interpretation in view of all the contrary evidence. We are thus forced to seek the basis of the mutant killer traits in the cytoplasm of the killer cell, mutation of kappa having already been indicated by these experiments as the most probable explanation for the altered killer character. 


\section{(f) Experiment 4}

If different kinds of killing are due to different kinds of kappa, then mutants that show two kinds of killing must contain two kinds of kappa. This crucial point can be put to a decisive test by applying to such killers techniques that reduce kappa concentration until some or most of the cells contain only one particle, then altering the conditions so that the residual particle multiplies and restores the full complement (Preer, 1946). These procedures, in effect, yield (in a killer animal and its descendants) a pure culture of kappa arising from a single particle. They thus make it possible to discover whether pure cultures of kappa derived from different kappa particles of one mixed killer animal may be of two diverse types. If so, then some of the resulting killer clones should be pure for one kind of killing and some for the other kind manifested by the ancestral mixed killer. On the other hand, if the two types of killing by such mixed killers are not due to two types of kappa, then each and every pure culture of kappa obtained by the dilution and restitution technique should again control both types of killing characteristic of the mixed killer ancestor.

The methods applicable to the original $5^{1}$ killer were developed by Sonneborn $(1946,1950 a)$. Animals are exposed to a temperature $\left(33^{\circ} 8^{\circ} \mathrm{C}\right.$. $)$ at which $5^{\mathrm{I}}$ kappa cannot increase, although the paramecia multiply rapidly. This approximately halves the amount of kappa per cell at each fission. After a number of fissions (depending on the original kappa concentration) an appreciable fraction of the cells have no kappa and most of the remainder have a single particle. At this stage, the animals are isolated and placed at $27^{\circ} \mathrm{C}$. with a limited amount of food daily (to keep fission rate at about one fission per day). Under these conditions, kappa multiplies much more rapidly than the paramecia, and those animals that retain one or more particles of kappa restore the full complement in the course of several days.

Specifically, these methods were applied by isolating vegetative killer animals singly into standard culture medium contained in test tubes in a $33.8^{\circ} \mathrm{C}$. water bath. At this temperature, animals multiply regularly and at maximal rate for eight or nine fissions, after which division tends to become progressively slower and irregular. At the ninth fission, the culture contained in each tube was thoroughly mixed, and approximately one-half of the total number of animals was removed by withdrawing half of the total volume of fluid. This volume was then replaced by an equivalent amount of fresh culture medium preheated to $33 \cdot 8^{\circ} \mathrm{C}$. Volumes withdrawn were distributed to depression slides and animals contained in them were counted, singly isolated, grown at $27^{\circ} \mathrm{C}$. and tested.

The result of applying this method to mutant $51 \mathrm{~m} 2$ fully confirmed the existence, in this killer, of two distinct types of kappa associated 
with two distinct types of killing. Of 193 animals isolated from $33.8^{\circ} \mathrm{C}$. at the ninth fission, $3^{\circ}$ produced cultures that were pure for Sp killing and resistance, but were sensitive to $5^{I}$ killers (in the latter respect differing from the $5 \mathrm{I} / \mathrm{m} 2$ parent), and $8 \mathrm{I}$ produced cultures that were non-killers but were resistant to $5^{1}$ killers and sensitive to Sp killers (in the latter respect also differing from the 5 I $m 2$ mutant). Thus, two distinct types were isolated, each differing from 5 I $m 2$ : one type of culture was pure for a kappa controlling $\mathrm{Sp}$ killing and resistance ; the other was pure for a kappa controlling resistance to $5^{\mathrm{I}}$, but little or no killing. The combination of these two kinds of kappa would indeed account for the phenotype of the 5 I $m 2$ stock: resistance to both $5^{I}$ and $\mathrm{Sp}$ killing and production of $\mathrm{Sp}$ paramecin.

As was to be expected, some of the isolates in this series yielded cultures exactly like the original 5 I $m 2$ killer. These would arise from animals containing at least one particle of each kind of kappa at the time they were removed from the high temperature. The frequency with which these occurred $(67)$ is in reasonable agreement with calculations based on the assumption of random segregation of the two kinds of kappa particles.

The results obtained with a second mixed killer, 5 I $m_{3}$, are in qualitative agreement with the results on 5 I $m 2$. Of 271 animals isolated at the ninth fission, 36 died, 23 were sensitive to both $5^{\text {I }}$ and $\mathrm{Sp}$ paramecin, $9^{2}$ were $5^{1}$ killers and sensitive to $\mathrm{Sp}$ paramecin, 74 were $\mathrm{Sp}$ killers and sensitive to $5^{1}$ paramecin, and 46 were mixed killers like $5 \mathrm{Im} 3$. Although the relative frequencics of these diverse classes are not in good agreement with the assumption of random segregation of two kinds of kappa, there can be no question as to the agreement with the main assumption of two distinct and separable types of kappa in the 5 I $m 3$ mutant. One type of kappa controls

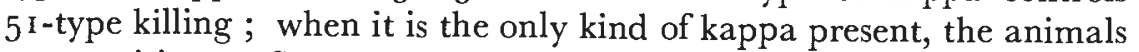
are sensitive to $\mathrm{Sp}$ paramecin. The other type of kappa resembles one of those extracted from 5 i $m_{2}$ : it controls Sp killing and when it is the only kind of kappa present, the animals are sensitive to $5^{\mathrm{I}}$ paramecin. When both kinds of kappa coexist in large quantities in the same animal, the latter kills in both ways and is resistant to both kinds of paramecin.

In contrast to the results on the mutant killers $5^{\text {I } m 2}$ and $5^{\text {I } m} 3$, the same methods applied to killers manifesting but a single type of killing ( $\mathrm{I}^{\mathrm{I}}, 5^{\mathrm{I}} \mathrm{mI}$ and $\left.5^{\mathrm{I}} \mathrm{m} 5\right)$, yielded but a single type of killer culture in each case : all of the isolates obtained from stock 5 I contained

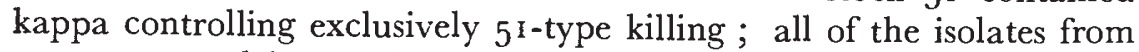
$5 \mathrm{I} m$ I possessed kappa determining only the Sp type of killing; and all of the isolates derived from $5^{\text {I }} \mathrm{m}_{5}$ contained kappa controlling exclusively the $5^{\text {I }} m_{5}$ phenotype.

Altogether, there thus seem to be but three clearly distinguishable types of kappa in the five mutants and the original $5^{I}$ killer stock. 
One kind, $5^{\mathrm{I}}$ kappa, controls the original $5^{\mathrm{I}}$ type of killing, and can multiply as rapidly as the cells that contain it. The second kind, Sp kappa, controls the spinner type of killing, and multiplies more slowly than $5^{\mathrm{I}}$ kappa. The third kind, $5_{\text {I }} r$ kappa, controls extremely weak $5 \mathrm{I}$ killing, or none at all, but it confers resistance to $5^{\mathrm{I}}$ paramecin and is able to multiply as rapidly as $5 \mathrm{I}$ kappa. Preliminary cytological studies indicate other characteristics of these kappas : the Sp kappa, with the possible exception of this type in $47 \mathrm{mI}$, seems to attain much higher concentration in the cell than does either $5 \mathrm{I}$ or $5^{\mathrm{I}} \mathrm{r}$ kappa ; and the frequency of double or multiple kappa particles may differ for different kinds of kappa.

Each of the diverse killers is characterised by the possession of one or two of these three kinds of kappa. The original $5^{1}$ killer has $5^{I}$ kappa only, $5_{\mathrm{I}} \mathrm{mI}$ has Sp kappa only, and $5_{\mathrm{I}} \mathrm{m}$ has $5^{\mathrm{I}} \mathrm{r}$ only; $5_{\mathrm{I} m 2}$ has Sp and $5_{5} \mathrm{I} r ; 5 \mathrm{I} m 3$ has Sp and $5 \mathrm{I}$. As set forth above in the discussion of experiment 2 (p. I 75), it is possible to construct mixed mutants from cultures each of which possesses only one type of kappa. Thus a culture resembling $5 \mathrm{I} \mathrm{m} 2$ was constructed by crossing $5^{\mathrm{I}} \mathrm{m} \mathrm{I}$ to $5^{\text {I } m 5}$ and selecting conjugant pairs that exchanged cytoplasm. In the same way, a culture resembling $5^{\mathrm{I}} \mathrm{m} 3$ was constructed by crossing $5^{\mathrm{I}} m \mathrm{I}$ to the original $5 \mathrm{I}$ killer.

These constructed killers further simulated the spontaneous mixed mutants in that the two kinds of kappa remained distinct and separable in them. Constructed types were cultivated for ten to twelve days and then subjected to the same procedure described above for isolating two kinds of kappa from $5 \mathrm{I} m 2$ and $5 \mathrm{I} m 3$. In each case, the two kinds of kappa that had been experimentally brought together were again experimentally separated from one another. The two kinds of kappa thus maintained their integrity even when in the same cell : each reproduced true to its own type, unaltered by the presence of the other.

Evidences previously cited (p. 174) led to the rejection of the possibilities that the kappas in the mutant stocks might have arisen de novo or by infection from an outside source. It was therefore concluded that these kappas were derived from the kappas of the two original killers, stocks 47 and $5 \mathrm{I}$. Among the mutant stocks there now exists at least two kinds of kappa that differ from both of the original kappas and that reproduce true to their new types. Each type controls a distinct kind of killing action. In sum, therefore, the physical basis of the killer character mutations has been found to lie in the mutation of kappa.

\section{DISCUSSION}

(I) The nature of kappa mutations.--The nature of the kappa mutations reported in this investigation is still largely a matter of speculation. If the specificity of kappa is due to one or more haptenelike groupings in the kappa particle, then a mutation might result from a change or loss of one or more of such groupings. Another 
possibility is suggested by the fact that in at least two of the five mutants examined, the original type of kappa is absent and two mutant types of kappa coexist. Since mutations of the killer character are detected very rarely, Sonneborn (1950 $b$ ) points out that the existence of even one double mutant among the five examined could hardly be coincidental. Since at least two of the five mutants analysed are double mutants and since Preer's (1950) data show that the kappa particles of his stock $G$ mutant killer is approximately one-half the size of that in the original $G$ killer, Sonneborn suggests that double mutations might arise as a result of a splitting of kappa into two different parts. There is as yet, however, no critical evidence from the present data which bears on this point.

(2) Kappa populations: equilibria and competition and their relation to the observed kappa conditions in the mutant killers. - Three of the five mutant killers have maintained two kinds of kappa, presumably in all of their descendants, for a period of at least two years. This observation immediately suggests that some sort of population equilibrium has been attained between various combinations of two kinds of kappa. The conclusion, however, is not necessarily justified, for there is another mechanism that could lead to the same result: viz. the mere coexistence of these two types of kappa within the cells could automatically perpetuate the combination by killing off all cells that lose either one of the two types of kappa. This would result in the appearance of typically affected animals within the culture. A simple way to obtain evidence as to whether maintenance of a mixed kappa population is due to such selective elimination of those animals that lose either kappa type, is to examine the culture for animals manifesting a response typical of sensitivity to either of the two kinds of paramecin in the culture. While it is often difficult to discover such sensitives in large mass cultures, their occurrence can readily be detected in small slide cultures of animals in nearly-starved condition.

Observations of this sort were carried out on two of the double mutants. In stock $5^{\mathrm{I}} \mathrm{m} 3$, animals were isolated singly into depression slides and grown at the rate of one fission per day until approximately 2000 animals were produced. At this stage, the animals are in proper nutritive condition to respond to paramecin action, and the presence of sensitive animals of either sort would be clearly manifested. Such observations were carried out on approximately 2000 descendants of each of 323 animals isolated from I I different 5 I $m 3$ subcultures at various times. Among the more than 60o,00o individuals examined, not one sensitive animal of either kind was found. As a check, all of the 323 cultures were then mixed with known sensitive animals and were shown to be typical $5^{\mathrm{I}} \mathrm{m}_{3}$ killers, i.e. producing both $5^{\mathrm{I}}$ and $\mathrm{Sp}$ paramecins. This failure on the part of $5 \mathrm{Im} 3$ clones to yield sensitive animals indicates that the combination of two kinds of kappa is not maintained, under such conditions, by selective elimination of animals that lose one kind of kappa. It would therefore seem that 
the two kinds of kappa are not subject to competitive replacement, but are maintained as a natural equilibrium of the two kappa populations.

The situation is somewhat different in the mutant $5 \mathrm{Im} 2$, which carries $\mathrm{Sp}$ and $5 \mathrm{I} r$ kappas : in this case, any animal that lost $\mathrm{Sp}$ kappa would be killed off by those that retained it, but any animal that lost 5 I $r$ kappa would probably survive, since the mixed killers (containing both Sp and $5^{\mathrm{I}} r$ kappas) produce little or no $5^{\mathrm{I}}$ paramecin. In this case, therefore, selective elimination could operate to destroy animals that had lost Sp kappa, but it would not operate effectively to destroy those that had lost $5^{\mathrm{I}} r$ kappa. The presence of the latter type of animal in cultures of $5 \mathrm{I} \mathrm{m} 2$ can be readily detected, however, by exposing these cultures to paramecin produced by the original $5^{\mathrm{I}}$ killer, for loss of $5^{\mathrm{I}} \mathrm{r}$ kappa makes the animals sensitive to $5^{\mathrm{I}}$ paramecin. This test was applied to some of the progeny of each of $2 \mathrm{II}$ single animal isolations from $5^{\mathrm{I}} \mathrm{m} 2$ clones. In no case were animals found that were sensitive to 5 I paramecin ; hence, no animals lacking $5 \mathrm{I} r$ kappa were present in the cultures of $5 \mathrm{I} \mathrm{m} 2$. Parts of these same cultures were also tested by mixing them with known sensitives; the tests showed that all of the $2 \mathrm{I}$ isolates produced Sp killing. Thus, like $5^{1} \mathrm{~m} 3$, the $5^{1} \mathrm{~m} 2$ mutant maintains a population equilibrium of two kinds of kappa under the conditions of culture employed.

The third possible combination of kappas, $5^{\mathrm{I}}$ and $5^{\mathrm{I} r}$, has not been analysed, since it has never been found as a spontaneous mutant. On the other hand, there are several lines of evidence indicating that such a kappa combination might behave differently from the combinations that have previously been examined. First, as has been stated, these two kappas are the most nearly identical of those studied ; yet, $5^{I} r$ is less active than $5^{I}$ kappa and may utilise more effectively the intracellular material required for its maintenance and reproduction. This could give 5 I $r$ a selective advantage in competition with $5 \mathrm{I}$ kappa. Secondly, 5 I $r$ kappa is known to have arisen from $5^{\mathrm{I}}$ kappa and established itself free from the latter in $5_{\mathrm{I}} \mathrm{m}_{5}$, a fact difficult to explain unless the $5 \mathrm{I} r$ kappa has the assumed selective advantage. Finally, the $5^{\mathrm{I}} \mathrm{m} 2$ mutant contains $\mathrm{Sp}$ and $5^{\mathrm{I}} \mathrm{r}$ kappas in equilibrium, without any $5^{\mathrm{I}}$ kappa being present. The absence of $5_{\mathrm{I}} \mathrm{kappa}$ is the more remarkable since it is known from the condition in 5 I $m 3$ that $\mathrm{Sp}$ and $5_{\mathrm{I}}$ kappas can establish an equilibrium. Hence, it would seem that the loss of $5^{\mathrm{I}}$ kappa from $5_{\mathrm{I}} \mathrm{m} 2$ is also to be interpreted as due to the selective advantage of $5^{1} r$ which displaced it. Taken together, these three lines of evidence warrant the tentative conclusion that $5 \mathrm{I}$ kappa is lost when $5 \mathrm{I} r$ kappa is present. This conclusion can, of course, be tested by crossing $5^{\text {I }}$ killers to $5^{\text {I } m 5}$ killers and obtaining cytoplasmic exchange between mates. This cross was actuaily made (p. 1 76), but the experiment was by no means conclusive since the mixed clones were followed for only eight fissions.

On the basis of the known equilibria established by $5 \mathrm{I}$ and $\mathrm{Sp}$ and by $5^{\mathrm{I}} r$ and $\mathrm{Sp}$, and on the indicated replacement of $5^{\mathrm{I}}$ by $5^{\mathrm{I} r}$ 
kappa when these two occur in the same cell, establishment of the mutants $5^{\mathrm{I}} \mathrm{m} 2,5^{\mathrm{I}} \mathrm{m} 3$ and $5^{\mathrm{I}} \mathrm{m} 5$ can readily be explained. The condition found in $5^{\text {I }} \mathrm{m}_{3}$ (coexistence of $5^{\mathrm{I}}$ and Sp kappas) would arise if a particle of $5^{I}$ kappa mutated to Sp kappa, for an equilibrium would eventually be established between these two kappas, and the remaining original $5 \mathrm{I}$ killers in the culture would be eliminated through the action of the $\mathrm{Sp}$ paramecin liberated by the mutants. Thus, the culture in which 5 I $m 3$ arose would transform in toto to a mutant killer culture. The condition found in $5^{1} \mathrm{~m} 2$, coexistence of $5^{\mathrm{I}} r$ and $\mathrm{Sp}$ kappas in the absence of $5^{\mathrm{I}}$ kappa, presents more of a problem. Here, however, after the origin of $5^{\mathrm{I} r} \mathrm{kappa}$, $5_{\mathrm{I}} \mathrm{kappa}$ would be expected to disappear. The Sp kappa present in the $5 \mathrm{I} m 2$ mutant could have arisen before or after the origin of $5^{\text {I }} \mathrm{k}$ kappa, or (on Sonneborn's hypothesis) at the same time. Once the mutant cell containing the kappa combination was established, the remaining $5^{\mathrm{I}}$ killers in the culture (as well as any cells which might have contained both $5^{\mathrm{I}}$ and $5^{\mathrm{I} r}$ kappas or $5^{\mathrm{I}} r$ alone) would have been eliminated by the action of Sp paramecin. Consequently, as in the case of 5 I $m 3$, the entire culture would eventually contain only 5 i $m 2$ animals. These conditions were, in fact, precisely those under which the $5^{1} \mathrm{~m} 2$ and $5_{5} \mathrm{I} m 3$ mutants were found.

Establishment of the $5^{\mathrm{I}} \mathrm{m}_{5}$ mutant is explained by a different set of conditions. Displacement of $5 \mathrm{I}$ kappa by $5^{\mathrm{I}} \mathrm{r}$ kappa has already been indicated. Thus, origin of $5^{1} r$ kappa within a $5^{1}$ killer animal woulc result in the loss of the original kappa and production of a $5^{\text {I } m} 5$ animal. Both $5^{\mathrm{I}}$ and $5_{\mathrm{I}} \mathrm{m}_{5}$ animals would be expected, however, to survive equally well within the same culture, since $\mathrm{Sp}$ paramecin is absent and since either type of animal is resistant to the paramecin of the other type. Such a culture would appear as a "weak" killer culture, however, since $5^{1} m 5$ animals produce little or no paramecin. Single animal isolations made from a weak killer culture of this sort would thus be expected to include some $5^{\text {I }} m_{5}$ animals. It was under exactly these conditions that the mutant was found.

At present, it is not possible to give a satisfactory explanation of mutant $5^{\mathrm{I} m \mathrm{I}}$ which contains only Sp kappa. There is no evident reason why $\mathrm{Sp}$ kappa should displace the ${ }^{\mathrm{I}} \mathrm{\text {kappa }}$ from which it arose, since $\mathrm{Sp}$ and $5 \mathrm{I}$ kappas were shown to establish an equilibrium in mutant $5 \mathrm{I} \mathrm{m}_{3}$. Further, if $5 \mathrm{I} \mathrm{mI}$ arose by mutation of $5 \mathrm{I}$ kappa to Sp kappa, there would be expected a strong selective advantage for the mixed kappa condition. It is possible, of course, that conditions prevailing in the stock bottle at the time the mutant arose were such as to operate against the establishment of an equilibrium between the two kinds of kappa. Finally, the fifth mutant, $47 \mathrm{mI}$, will not be discussed in view of the fact that so little is known about the kappa from which this mutant arose. The apparent difference in interaction between the $5 \mathrm{I}$ and $\mathrm{Sp}$ kappas in $47 \mathrm{mI}$, and the $5^{\mathrm{I}}$ and Sp kappas in $5^{\mathrm{I}} \mathrm{m} 3$ (as inferred from table 3 ) could be due 
to the difference in genotype (47 and 51 ) with which this kappa combination is associated.

\section{SUMMARY}

I. The purpose of the present investigation was to determine whether changes in nuclear genes or cytoplasmic factors or both were responsible for the production of five mutations of the killer character in certain strains of Paramecium aurelia.

2. Four of these mutations were derived from cultures of the killer stock $5 \mathbf{I}$; the fifth arose in killer stock 47 . In all cases, so far as is known, the mutations were of spontaneous origin.

3. The five mutants differed from the parent stocks in either the type of killing or in the strength of killing as measured by the numbers of sensitive animals killed in a given period of time. These differences were maintained throughout vegetative and sexual reproduction.

4. Two general types of killing action were manifested by the mutant stocks. The first type was identical with that produced by the original $5 \mathrm{I}$ parent. The second type differed strikingly from stock $5 \mathrm{I}$, causing affected animals to spin violently prior to death.

5. Animals whose killing was of one type were resistant to killing action of that type but not to killing action of the other type. Animals whose killing was of both types were completely resistant to both types.

6. A series of crosses demonstrated that: (a) the presence of a "killer" gene in each mutant stock was necessary for determination of mutant killing, $(b)$ the presence of a kappa-type of cytoplasmic factor in each mutant stock was also necessary for determination of the killer character, $(c)$ in the presence of the killer gene, $K$, from the original stock $5 \mathrm{I}$, the mutant killer characters were maintained, $(d)$ in the presence of the killer gene from each mutant stock, the original $5^{\mathrm{I}}$ killer character was preserved, $(e)$ the killer gene in each mutant stock must then be effectively identical with the $K$ gene in the original stocks from which they were derived.

7. The results expected if the mutant killing had been due to mutation of the $K$ gene or to some other gene influencing the killer character were not obtained. On the contrary, the results showed that the mutants did not differ from the original in any genes affecting this trait.

8. Three distinct kinds of kappa were separated out from the mutants and obtained in pure form in killer cells. This was accomplished by dilution of the kappa concentration in the mutants to a single particle per animal, followed by slow growth of the animal to permit the particle to restore the maximal kappa concentration.

9. Three explanations of the origin of the different kappas were considered : de novo origin, infection, and mutation of the kappa originally present. As the first two could be effectively excluded, it was concluded that the physical basis of the killer character mutations was mutation of the kappas in the original stocks. 
Io. One of the three types of kappa found in the mutant stocks

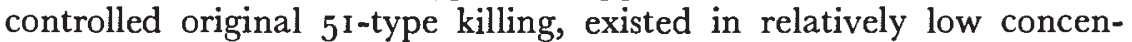
tration within the killer animal, and multiplied as rapidly as the cell itself.

A second type of kappa, Sp, controlled the spinner type of killing, was present in high concentration in the killers, and multiplied more slowly than $5 \mathrm{I}$ kappa.

The third kind, $5^{\text {I }}$ kappa, controlled extremely weak $5^{\text {I }}$ killing, or none at all, but it conferred resistance to $5^{\mathrm{I}}$ paramecin. This kappa was able to multiply as rapidly as $5 \mathrm{I}$ kappa and its concentration per cell more nearly resembled that of 51 kappa than the Sp kappa.

II. Among the five mutant stocks, 5I $\mathrm{I}$ I was pure for Sp kappa, and $5^{\mathrm{I} m} 5$ was pure for $5^{\mathrm{I} r}$ kappa. The remaining three mutants each contained two kinds of kappa : $5^{\mathrm{I}} \mathrm{m}_{3}$ contained $5^{\mathrm{I}}$ and $\mathrm{Sp}$ kappas, $5^{\mathrm{I} m 2}$ contained $5^{\mathrm{I} r}$ and Sp kappas, and $47 \mathrm{mI}$ contained $5^{\mathrm{I}}$ and Sp kappas. The latter two mutants thus not only contained two kinds of kappa but both differed from the original kappa.

12. Within a cell and its progeny, under standard conditions, certain combinations of two kinds of kappa (viz. Sp and $5^{1}$, or $\mathrm{Sp}$ and $5 \mathrm{I} r$ ) attain and maintain an equilibrium population. On the other hand, there are indications that 5 I $r$ kappa possesses a selective advantage over $5 \mathrm{I}$ kappa, displacing the latter from a cell when these two types occur together. These relations among the kappa types provide a plausible explanation for the establishment and detection of mutants $5^{\mathrm{I} m} \mathrm{~m}, 5^{\mathrm{I}} \mathrm{m}_{3}$ and $5_{\mathrm{I}} \mathrm{m}_{5}$.

\section{REFERENCES}

DELAMATER, E. D. 1948.

Basic fuchsin as a nuclear stain for fungi.

Mycologia, 4o, 423-429.

DIPPELL, RUTH V. 1948.

Mutations of the killer plasmagene, kappa, in variety 4 of Paramecium aurelia.

Amer. Nat., 82, 43-50.

GOLDSTEIN, L. I 949 .

Contribution a l'étude de la sensibilité héréditaire au gaz carbonique chez la

Drosophile. Mise en évidence d'une forme nouvelle du génoide.

Bulletin Biologique de la France et de la Belgique, 83, I77-1 88.

PREER, J. R. 1946.

Some properties of a genetic cytoplasmic factor in Paramecium.

P.N.A.S., 32, 247-253.

PREER, J. R. I $1948 a$.

A study of some properties of the cytoplasmic factor, "kappa," in Paramecium curelia, variety 2.

Genetics, 33, 349-404.

PREER, J. R. I $948 b$.

Microscopic bodies in the cytoplasm of " killers" of Paramecium aurelia and evidence for the identification of these bodies with the cytoplasmic factor, kappa.

Genetics, 33, 625. 
PREER, J. R. I950.

Microscopically visible bodies in the cytoplasm of the "killer" strains of Paramecium aurelia.

Genetics, 35, 344-362.

RHOADES, M. M. 1946.

Plastid mutations.

Cold Spring Harbor Symp. Quant. Biol., II, 202-207.

SONNEBORN, T. M. I939.

Paramecium aurelia : mating types and groups; lethal interactions; determination and inheritance.

Amer. Nat., 73, 390-413.

SONNEBORN, T. M. I940.

The relation of macronuclear regeneration in Paramecium aurelia to macronuclear structure, amitosis and genetic determination.

Anat. Rec., 78, 53-54

SONNEBORN, T. M. 1943 .

Gene and cytoplasm. I. The determination and inheritance of the killer character in variety 4 of $P$. aurelia. II. The bearing of the determination and inheritance of characters in $P$. aurelia on the problems of cytoplasmic inheritance, Pneumococcus transformations, mutations and development.

P.N.A.S., 29, 329-343.

SONNEBORN, T. M. I944.

Exchange of cytoplasm at conjugation in Paramecium aurelia, variety 4 .

Anat. Rec., 89, 49 .

SONNEBORN, T. M. 1945 .

The dependence of the physiological action of a gene on a primer and the relation of primer to gene.

Amer. Nat., 79, 318-329.

SONNEBORN, T. M. 1946 .

Experimental control of the concentration of cytoplasmic factors in Paramecium. Cold Spring Harbor Symp. Quant. Biol., II, 236-255.

SONNEBORN, T. M. I947a.

Recent advances in the genetics of Paramecium and Euplotes.

Advances in Genetics, $1,263-35^{8}$.

SONNEBORN, T. M. I $1947 b$.

Developmental mechanisms in Paramecium.

Growth Symposium, II, 291-307.

SONNEBORN, T. M. 1948 .

Symposium on plasmagenes, genes and characters in Paramecium aurelia. Introduction. Amer. Nat., 82, 26-34.

SONNEBORN, T. M. I95Oa.

Methods in the general biology and genetics of Paramecium aurelia.

7. Exp. Zool., II3, 87-148.

SONNEBORN, T. M. I95Ob.

The cytoplasm in heredity.

Heredity, 4, I I -36.

SONNEBORN, T. M., AND DIPPELL, RUTH v. 1946.

Mating reactions and conjugation between varieties of Paramecium aurelia in relation to conceptions of mating type and variety.

Physiol. Zool., I9, I-18. 


\section{Plate I}

\section{Explanation of rigures}

FIGs. $(a)$ to $(i)$.-Photomicrographs of the morphological changes undergone by sensitive animals exposed to paramecins of three different killer stocks. Figs. $(a),(b),(f),(g)$, $(h)$ and $(i)$ : living animals. Figs. $(c),(d)$ and $(e)$ : fixed and stained preparations. (The large, deeply staining body is the macronucleus.) Background has been blocked out in all figures except $(f)$. Approximate magnification : $\times 200$.

\section{Effect of stock $5 \mathrm{I}$ paramecin}

(a) and (b) early stages of effect, showing small blisters that appear and enlarge on body surface.

$(c)$ and $(d)$ development of the large posterior " hump," the most characteristic of the responses by sensitives to 5 I paramecin.

(e) final stage of killing effect. Animals decrease in size to at least one-half of that shown in $(a)$ and assume a pear-shaped to spherical form prior to death.

\section{Effect of stock 47 paramecin}

( $f$ ) characteristic response : development of a single large vacuole in posterior part of body. (This photograph was obtained by Dr T. M. Sonneborn prior to loss of killer character by stock 47 .)

\section{Effect of mutant stock $5 \mathrm{I}_{\mathrm{I}}$ paramecin}

$(g)$ and $(h)$ vacuolation of sensitives subjected to $5 \mathrm{I} m \mathrm{I}$ paramecin.

(i) final stage of killing action, characterised by paralysis or formation of huge blister on body surface into which body contents may subsequently empty. 
